Revta brasil. Bot., São Paulo, V.23, n.4, p.359-370, dez. 2000

\title{
Briófitas de campos rupestres da Chapada Diamantina, Estado da Bahia, Brasil
}

\author{
CID JOSÉ PASSOS BASTOS ${ }^{1,3}$, OLGA YANO ${ }^{2}$ e SILVANA B. VILAS BÔAS- BASTOS ${ }^{1}$
}

(recebido em 28 de julho de 1999; aceito em 17 de maio de 2000)

\begin{abstract}
Bryophytes of "campos rupestres" of Chapada Diamantina, state of Bahia, Brazil). In this taxonomic survey 65 taxa were found in "campos rupestres" of Bahia, 41 belonging to Bryophyta distributed into 19 genera and 11 families, and 24 belonging to Hepatophyta distributed into 15 genera and nine families. Of these, 23 (nine mosses and 14 hepatics) are new records for the state. Most of the taxa seem to be restricted to the Chapada Diamantina and have not been found in other sites in the state.

RESUMO - (Briófitas de campos rupestres da Chapada Diamantina, Estado da Bahia, Brasil). Nesse levantamento, foram encontrados 65 táxons infragenéricos nos campos rupestres da Bahia, sendo 41 pertencentes à divisão Bryophyta, distribuídos em 19 gêneros e 11 famílias, e 24 à divisão Hepatophyta distribuídos em 15 gêneros e nove famílias. Desses, 23 (nove musgos e 14 hepáticas) são novas citações para o estado. A maioria desses táxons parece ter distribuição restrita à região da Chapada Diamantina, não tendo sido encontrados em outras regiões do estado.
\end{abstract}

Key words - Bryophyta, Hepatophyta, "Campos Rupestres", Bahia, Brazil

\section{Introdução}

No Estado da Bahia, os campos rupestres ocorrem nas áreas mais elevadas da Serra do Espinhaço, a principal cadeia montanhosa do Planalto Central, constituída por dois blocos principais: a Cadeia do Espinhaço, em Minas Gerais, e a Chapada Diamantina, na Bahia. A Serra do Espinhaço é composta, em sua maior parte, por arenitos do Pré-Cambriano e de rochas metamórficas, formada na parte leste pelo Escudo Cristalino Brasileiro, tendo a sua formação ocorrida a partir do Paleozóico. A Serra do Espinhaço constitui-se num complexo geomorfológico em que diversos tipos de rochas (arenitos metamórficos, quartzitos, conglomerados e rochas ígneas) contribuem para a formação de um relevo muito acidentado, resultando num complexo de formações vegetais características, determinado também pelas condições climáticas locais (Harley 1995a).

As briófitas estão bem representadas nesse ambiente, principalmente nas matas de neblinas da Chapada Diamantina e nas florestas de galeria, ao longo dos rios que cortam essa região. A primeira contribuição efetiva para o conhecimento da brioflora dessa área foi dada por Harley (1995b), que compi-

1. Universidade Federal da Bahia, Instituto de Biologia, Departamento de Botânica. Campus de Ondina, 40170-280 Salvador, Bahia, Brasil

2. Instituto de Botânica, Caixa Postal 4005, 01061-970 São Paulo, SP, Brasil.

3. Autor para correspondência: cjpbasto@ufba.br lou os dados de briófitas coletadas no Pico das Almas. Mais recentemente, contribuição adicional foi dada por Bastos et al. (1998), que realizaram um levantamento de briófitas em floresta de galeria, nas proximidades da cidade de Lençóis, onde foram identificadas 31 espécies, sendo 27 de musgos e quatro de hepáticas. Registros isolados podem ser encontrados em Yano (1981, 1984, 1989, 1995) e em Yano \& Bastos (1994).

Poucos têm sido os trabalhos sobre campos rupestres brasileiros, podendo-se citar apenas Yano (1987) para a Serra do Cipó e Yano \& Carvalho (1995) para a Serra da Piedade, ambos em Minas Gerais. O objetivo deste trabalho é contribuir para o melhor conhecimento das briófitas dessa formação vegetal.

\section{Material e métodos}

Foram analisados exemplares procedentes de campos rupestres da Chapada Diamantina, depositados no Herbário Científico do estado "Maria Eneyda P. Kaufmann Fidalgo", do Instituto de Botânica de São Paulo (SP), no Herbário Alexandre Leal Costa, do Instituto de Biologia da Universidade Federal da Bahia (ALCB) e alguns do Herbário do Centro de Pesquisas do Cacau (CEPEC).

O clima dessa região é seco a subúmido e semi-árido, com temperatura média anual de $22^{\circ} \mathrm{C}$, com máxima de $27,2{ }^{\circ} \mathrm{C}$ e mínima de $17,8^{\circ} \mathrm{C}$. O período chuvoso vai de novembro a janeiro, com pluviosidade média anual de 600 a 1000 mm (CEI, 1994).

As identificações basearam-se nas publicações de Lindenberg (1844), Fulford (1963, 1966), Stotler (1969), Schuster (1980), Crum (1984, 1987), Yano et al. (1985), Frahm (1991), Yano (1992), Gradstein (1994), Sharp et al. (1994) e Churchill \& Linares (1995). O arranjo sistemático para as famílias baseou-se em Vitt (1984) para as Bryophyta e em Schuster (1984) para as 
Hepatophyta. Nos resultados, os asteriscos indicam novas citações para o estado da Bahia.

\section{Resultados}

Foram identificados 65 táxons infragenéricos, sendo 41 pertencentes à divisão Bryophyta, distribuídos em 19 gêneros e 11 famílias, e 24 à divisão Hepatophyta, distribuídos em 15 gêneros e nove famílias. Dentre as Bryophyta, as famílias mais bem representadas foram Dicranaceae, com nove táxons, Leucobryaceae, com 10 táxons e Sphagnaceae, com seis táxons. Dentre as Hepatophyta destacaram-se Lejeuneaceae, com seis táxons e Lepidoziaceae e Plagiochilaceae com cinco táxons cada uma.

Destes 65 táxons, nove Bryophyta e 14 Hepatophyta são novas citações para o estado da Bahia.

\section{I - Bryophyta}

\section{Sphagnaceae}

Sphagnum capillifolium (Ehrh.) Hedw., Fund. Musc. 2: 86. 1782.

Descrição e ilustração: Crum (1984).

Material examinado: BAHIA: Morro do Chapéu, 3/4/1976, D.M. Vital 6051 (SP 135074); Serra de Mucugê, 2/11/1973, A.L. Costa s.n. (ALCB 18176); município de Abaíra, Campo de Ouro Fino (baixo), $13^{\circ} 15^{\prime}$ 'S e $41^{\circ} 54$ '"W, alt. 1600-1700 m, 18/1/1992, D.J.N. Hind \& R.F. Queiroz s.n. (SP 284203).

Comentários: encontrada crescendo sobre o solo e rochas úmidas. Na Bahia, restrita à Chapada Diamantina, não tendo sido encontrada em outra região do estado.

Sphagnum harleyi Crum, J. Hattori Bot. Lab. 63: 80. 1987.

Descrição e ilustração: Crum (1987).

Material examinado: BaHIa: Abaíra, Campo do Cigano, $13^{\circ} 15^{\prime} \mathrm{S}$ e $41^{\circ} 55^{\prime} \mathrm{W}$, alt. $1780 \mathrm{~m}, 14 / 2 / 1992$, R.M. Harley et al. (SP 284204); Mucugê, Unidade de manejo sustentável do projeto Sempre Viva, 18/3/1998, M.T.S. Stradmann s.n. (ALCB 37737).

Comentários: encontrada crescendo sobre rochas e paredões úmidos. Na Bahia, restrita à Chapada Diamantina, não tendo sido encontrada em outra região do estado.
Sphagnum longistolo C. Muell. in Warnst., Hedwigia 36: 169. 1897.

Descrição e ilustração: Yano et al. (1985).

Material examinado: BaHIA: Morro do Chapéu, s.d., G.C. Pinto s.n. (ALCB 18676). Morro do Chapéu, 4/4/1976, D.M. Vital 6052 (SP 135075).

Comentários: encontrada crescendo sobre rochas úmidas, na margem de rio. Referida também, para Jacobina (Yano et al. 1985), sem especificação de ecossistema.

Sphagnum magellanicum Brid., Musc. Rec. 2: 24. 1798.

Descrição e ilustração: Crum (1984); Yano et al. (1985).

Material examinado: BAHIA: Abaíra, Campo de Ouro Fino (alto), $13^{\circ} 15^{\prime} \mathrm{N}$ e $41^{\circ} 54^{\prime} \mathrm{W}$, alt. $1700 \mathrm{~m}$, 19/1/1992, D.J.N. Hind \& R.F. Queiroz s.n. (SP 284205).

Comentários: encontrada crescendo sobre rochas úmidas. Na Bahia, restrita à Chapada Diamantina, não tendo sido encontrada em outra região do estado.

Sphagnum papillosum Lindb., Acta Soc. Sci. Fenn. 10: 280. 1872. (1985).

Descrição e ilustração: Crum (1984); Yano et al.

Material examinado: BAHIA: Mucugê, 6/6/1981, M.C. Amaral s.n. (SP 170468).

Comentários: encontrada crescendo sobre solo de várzea. Na Bahia, restrita à Chapada Diamantina, não tendo sido encontrada em outra região do estado.

Sphagnum recurvum P. Beauv., Prodr. 88. 1805.

Descrição e ilustração: Crum (1984); Yano et al. (1985).

Material examinado: BAHIA: Abaíra, serra ao sul do Riacho da Taquara, $13^{\circ} 15^{\prime} \mathrm{S}$ e $41^{\circ} 55^{\prime} \mathrm{W}$, alt. 1800-1900 m, 27/1/1992, D.J. Hind \& R.F. Queiroz s.n. (SP 284206).

Comentários: encontrada crescendo sobre solo arenoso e rochas úmidas. Na Bahia, restrita à Chapada Diamantina, não tendo sido encontrada em outra região do estado. 
2. Polytrichaceae

*Polytrichum brasiliense Hampe, Vid. Midd. Naturh. For Kjoebenh. ser. 3,4: 53. 1872.

Descrição e ilustração: Farias (1984); Oliveira e Silva (1998).

Material examinado: BAHIA: Abaíra, Mata da Serra do Rei, $14^{\circ} 16^{\prime} \mathrm{S}$ e $41^{\circ} 54^{\prime} \mathrm{W}$, alt. $1550-1650 \mathrm{~m}$, 17/2/1992, R.M. Harley et al. (SP 284234).

Comentários: encontrada crescendo entre rochas, em brejo. Até o momento, não encontrada em outra região do estado.

Polytrichum juniperinum Willd. ex Hedw., Spec. Musc. 89. 1801.

Descrição e ilustração: Bartram (1949).

Material examinado: BAHIA: Palmeiras, Pai Inácio, BR 242, km 232, ca. $15 \mathrm{~km}$ ao $\mathrm{N}$ de Palmeiras, alt. $1000-1200$ m, 24/12/1979, S.A. Mori \& F.P. Benton 13232 (ALCB 18839); Abaíra, Campo do Cigano, $13^{\circ} 15^{\prime}$ 'S e $41^{\circ} 55^{\prime} \mathrm{W}$, alt. $1780 \mathrm{~m}, 14 / 2 / 1992$, R.M. Harley et al. (SP 284233; SP 284234); alt. 1800 m, 29/1/1992, M.T.V.A. Campos s.n. (SP 284235).

Comentários: encontrada crescendo sobre rochas e lajedos areníticos. Na Bahia, restrita à Chapada Diamantina, não tendo sido encontrada em outra região do estado.

\section{Orthotrichaceae}

Macromitrium punctatum (Hook. \& Grev.) Brid., Bryol. Univ. 1: 739. 1826.

Descrição e ilustração: Sharp et al. (1994).

Material examinado: BAHIA:Abaíra, Campo do Ouro Fino (baixo), $13^{\circ} 15^{\prime} \mathrm{S}$ e $41^{\circ} 54^{\prime} \mathrm{W}$, alt. 1600 1700 m, 25/1/1992, D.J.N. Hind \& R.F. Queiroz s.n. (SP 284212).

Comentários: encontrada crescendo sobre rocha e tronco de árvore. Encontrada, também, em floresta de galeria, floresta ombrófila e floresta estacional.

Schlotheimia rugifolia (Hook. \& Grev.) Schwaegr., Spec. Musc. Suppl. 2(1): 150. 1824.

Descrição e ilustração: Sharp et al. (1994).

Material examinado: BAHIA: Morro do Chapéu, Ferro Doido, 18/5/1975, D.V. Martins s.n. (ALCB 29241); município de Abaíra, Campo do Ouro Fino (baixo), $13^{\circ} 15^{\prime} \mathrm{S}$ e $41^{\circ} 54^{\prime} \mathrm{W}$, alt. $1600-1700 \mathrm{~m}$, 18/1/1992, D.J.N. Hind \& R.F. Queiroz s.n. (SP 284213); Campo do Ouro Fino (alto), alt. 1700 m,
19/1/1992, D.J.N. Hind \& R.F. Queiroz s.n. (SP 284214); Riacho da Taquara, $13^{\circ} 15^{\prime} \mathrm{S}$ e $41^{\circ} 55^{\prime} \mathrm{W}$, alt. $1670 \mathrm{~m}, 14 / 2 / 1992$, B. Stannard et al. s.n. (SP 284215); Campo de Ouro Fino (baixo), $13^{\circ} 15^{\prime} \mathrm{S}$ e 41 ${ }^{\circ} 54^{\prime}$ W, alt. 1600-1700 m, 18/1/1992, D.J.N. Hind \& R.F. Queiroz s.n (SP 284216); Rio Mucugezinho, 30/8/1994, M.T. Stradmann 15 (ALCB 28270); Mucugê, Unidade de manejo sustentável do projeto Sempre Viva, 18/3/1998, M.T. Stradmann s.n (ALCB 36693).

Comentários: encontrada crescendo sobre tronco de árvore, tronco em decomposição e sobre rocha. Espécie bastante comum na Chapada Diamantina, tendo sido encontrada, também, em floresta de galeria.

4. Bryaceae

Bryum argenteum Hedw., Spec. Musc. 181. 1801.

Descrição e ilustração: Sharp et al. (1994).

Material examinado: BaHIA: Abaíra, Campo do Cigano, $13^{\circ} 15^{\prime} \mathrm{N}, 41^{\circ} 55^{\prime} \mathrm{W}$, alt. $1780 \mathrm{~m}, 14 / 2 / 1992$, R.M. Harley et al. s.n (SP 284226); Campo do Ouro Fino (alto), $13^{\circ} 15^{\prime} \mathrm{S}$ e $41^{\circ} 54^{\prime} \mathrm{W}$, alt. $1800 \mathrm{~m}$, 19/1/1992, D.J.N. Hind \& R.F. Queiroz s.n. (SP 284227); Palmeiras, Chapadinha, 3/9/1994, M. Fonseca s.n.(ALCB 18926).

Comentários: encontrada crescendo sobre rocha e solo. Bastante comum na Bahia, ocorrendo em áreas urbanas e em caatinga.

Bryum coronatum Schwaegr., Spec. Musc. Suppl. 1(2): 103. 1816.

Descrição e ilustração: Sharp et al. (1994).

Material examinado: BaHIA: Mucugê, Unidade de manejo sustentável do projeto Sempre Viva, 17/3/1998, M.T.S. Stradmann s.n. (ALCB 37727).

Comentários: encontrada crescendo sobre o solo. Comum na Bahia, ocorrendo em restinga, cerrado e áreas antrópicas.

*Bryum paradoxum Schwaegr., Spec. Musc. Suppl. 3(1): 224a. 1827.

Descrição e ilustração: Ochi (1980).

Material examinado: BAHIA: Abaíra, Campo do Cigano, $13^{\circ} 15^{\prime} \mathrm{S}$ e $41^{\circ} 55^{\prime} \mathrm{W}$, alt. $1780 \mathrm{~m}, 14 / 2 / 1992$, R.M. Harley et al. s.n. (SP 284228). 
Comentários: encontrada crescendo sobre o solo. Até o momento, não encontrada em outra região do estado.

\section{Rhizogoniaceae}

Pyrrhobryum spiniforme (Hedw.) Mitt., J.Linn. Soc. Bot. 10: 174. 1868.

Descrição e ilustração: Sharp et al. (1994).

Material examinado: BAHIA: Rio de Contas, Pico das Almas, vertente leste, alt.1500-1550 m, 27/9/1988, R.M. Harley 26631 (ALCB 28238); Abaíra, Campo do Cigano, $13^{\circ} 15^{\prime} \mathrm{S}$ e $41^{\circ} 55^{\prime} \mathrm{W}$, alt. 1780 m, 14/2/1992, R.M. Harley et al. s.n. (SP 284231); Mucugê, 20/9/1998, M.L. Guedes s.n. (ALCB 37741).

Comentários: encontrada crescendo sobre o solo e tronco de árvore. Muito comum na Chapada Diamantina; encontrada, também, em floresta de galeria.

\section{Sematophyllaceae}

Aptychopsis pyrrhophylla (C. Muell.) Wijk \& Marg., Taxon 8: 71. 1959.

Descrição: Müller (1851, como Нypnum pyrrhophyllum C. Müll.).

Material examinado: BaHiA: Rio de Contas, Pico das Almas, vertente leste, $13^{\circ} 31^{\prime}$ S e $41^{\circ} 58^{\prime} \mathrm{W}$, alt. 1500-1550 m, 27/11/1988, R.M. Harley 26634 (ALCB 28246; CEPEC 50116).

Comentários: encontrada crescendo sobre tronco de árvore. Na Bahia, restrita à Chapada Diamantina, não tendo sido encontrada em outra região do estado.

*Colobodontium vulpinum (Mont.) Churchill \& Buck, Bibl. J.J. Triana 12(2): 759. 1995.

Descrição e ilustração: Churchill \& Linares (1995).

Material examinado: BAHIA: Pai Inácio, 24/8/1996, M.T.S. Stradmann s.n. (ALCB 29468).

Comentários: encontrada crescendo sobre rocha, na margem de um córrego. Até o momento, não encontrada em outra região do estado.

Sematophyllum subpinnatum (Brid.) Britt., Bryologist 21: 28. 1918.

Descrição e ilustração: Sharp et al. (1994).
Material examinado: BAHIA: Abaíra, Campo de Ouro Fino (baixo), $13^{\circ} 15^{\prime} \mathrm{S}$ e $41^{\circ} 54^{\prime} \mathrm{W}$, alt. 1600 1700 m, 18/1/1992, D.J.N. Hind \& R.F. Queiroz s.n. (SP 284184).

Comentários: encontrada crescendo sobre raízes expostas. Muito comum na Bahia, ocorrendo em floresta de galeria, cerrado, floresta ombrófila, floresta estacional e restinga arbórea.

\section{Meteoriaceae}

Squamidium brasiliensis (Hornsch.) Broth., Naturl. Pflazenfam. 1(3): 809. 1906.

Descrição e ilustração: Allen \& Crosby (1986).

Material examinado: BAHIA: Rio de Contas, Pico das Almas, vertente leste, Campo do Queiroz, perto da trilha da fazenda Silvina, $13^{\circ} 32^{\prime} \mathrm{S}$ e 41 $57^{\prime}$ 'W, alt. 1500 m, 27/11/1988, R.M. Harley 26643 (ALCB 28249; CEPEC 55125); Abaíra, Campo de Ouro Fino (baixo), $13^{\circ} 15^{\prime} \mathrm{S}$ e $41^{\circ} 54^{\prime} \mathrm{W}$, alt. 1600-1700 m, 18/1/1992, D.J.N. Hind \& R.F. Queiroz s.n. (SP 284238).

Comentários: encontrada crescendo sobre rocha e tronco de árvore. $\mathrm{Na}$ Bahia, restrita à Chapada Diamantina, não tendo sido encontrada em outra região.

\section{Phyllogoniaceae}

Phyllogonium viride Brid., Bryol. Univ. 2: 673. 1827.

Descrição e ilustração: Buck (1998).

Material examinado: BAHIA: Abaíra, Campo de Ouro Fino (baixo), $13^{\circ} 15^{\prime} \mathrm{S}$ e $41^{\circ} 54^{\prime} \mathrm{W}$, alt. 16001700 m, 18/1/1992, D.J.N. Hind \& R.F. Queiroz s.n (SP 284229; SP 284230).

Comentários: encontrada crescendo sobre tronco de árvore. Comum na Bahia, ocorrendo em floresta ombrófila e floresta estacional.

\section{Calymperaceae}

Syrrhopodon prolifer Schwaegr. var. prolifer, Spec. Musc. Suppl. 2(2): 99. 1827.

Descrição e ilustração: Sharp et al. (1994).

Material examinado: BAHIA: Abaíra, Campo e Ouro Fino (baixo), $13^{\circ} 15^{\prime} \mathrm{S}$ e $41^{\circ} 54^{\prime} \mathrm{W}$, alt. 16001700 m, 18/1/1992, D.J.N. Hind \& R.F. Queiroz s.n. (SP 284236). 
Comentários: encontrada crescendo na base do tronco de árvore. Comum na Bahia, ocorrendo em cerrado e floresta de galeria.

* Syrrhopodon prolifer Schwaegr. var. scaber (Mitt.) Reese, Flora Neotropica 58: 13. 1993.

Descrição e ilustração: Reese (1993).

Material examinado: BAHIA, Mucugê, 20/9/1998, M.L. Guedes s.n. (ALCB 37740).

Comentários: encontrada crescendo em fendas de rocha. Até o momento, não encontrada em outra região do estado.

* Syrrhopodon prolifer Schwaegr. var. tenuifolius (Sull.) Reese, Bryologist 81: 199. 1978.

Descrição e ilustração: Sharp et al. (1994).

Material examinado: BAHIA: Abaíra, Campo do Cigano, $13^{\circ} 15^{\prime} \mathrm{S}$ e $41^{\circ} 55^{\prime} \mathrm{W}$, alt. $1780 \mathrm{~m}, 14 / 2 / 1992$, R.M. Harley et al. s.n. (SP 284237).

Comentários: encontrada crescendo sobre o solo e em fendas de rochas. Até o momento, não encontrada em outra região do estado.

\section{Dicranaceae}

Bryohumbertia filifolia (Hornsch.) J.-P. Frahm, Cryptogamie, Bryol. Lichénol. 3: 366. 1982.

Descrição e ilustração: Frahm (1991).

Material examinado: BAHIA: Mucugê, Unidade de manejo sustentável do projeto Sempre Viva, 18/3/1998, 29/5/1998, M.T.S. Stradmann s.n. (ALCB 37738; ALCB 37729).

Comentários: encontrada crescendo sobre o solo. Na Bahia, restrita à Chapada Diamantina, não tendo sido encontrada em outra região do estado.

Campylopus arctocarpus (Hornsch.) Mitt. var. caldense (Ängstr.) J.-P. Frahm, Flora Neotropica Monograph 54: 55. 1991.

Descrição e ilustração: Frahm (1991)

Material examinado: BAHIA: Abaíra, Campo de Ouro Fino (baixo), $13^{\circ} 15^{\prime} \mathrm{S}$ e $41^{\circ} 54^{\prime} \mathrm{W}$, alt. 16001700 m, 18/1/1992, D.J.N. Hind \& R.F. Queiroz s.n. (SP 284207).

Comentários: encontrada crescendo sobre o solo. Na Bahia, restrita à Chapada Diamantina, não tendo sido encontrada em outra região do estado.
Campylopus dichrostis (C. Muell.) Par. in Broth., Naturl. Pflanzenfam. 1(3): 333. 1901.

Descrição e ilustração: Frahm (1991).

Material examinado: BAHIA: Mucugê, Unidade de manejo sustentável do projeto Sempre Viva, 18/3/1998, M.T.S. Stradmann s.n. (ALCB 37734).

Comentários: encontrada crescendo sobre rocha. Na Bahia, restrita à Chapada Diamantina, não tendo sido encontrada em outra região do estado.

Campylopus introflexus (Hedw.) Brid., Mant. Musc. 72. 1819.

Descrição e ilustração: Frahm (1991).

Material examinado: BAHIA: Morro do Chapéu, Ferro Doido, 18/5/1975, D.V. Martins s.n. (ALCB 18152).

Comentários: encontrada crescendo sobre rochas úmidas. Na Bahia, restrita à Chapada Diamantina, não tendo sido encontrada em outra região do estado.

Campylopus julaceus Jaeg., Ber. Thätigk. St. Gall. Naturwiss. Ges. 1879-1878: 384. 1880.

Descrição e ilustração: Frahm (1991).

Material examinado: BAHIA: Morro do Chapéu, Ferro Doido, 18/5/1975, D.V. Martins s.n. (ALCB 38081); Mucugê, Unidade de manejo sustentável do projeto Sempre Viva, 19/3/1998, M.T.S. Stradmann s.n. (ALCB 37728); Cachoeira do Tiburtino, Rio Cumbuca, 15/3/1998, M.T.S. Stradmann s.n. (ALCB 37730).

Comentários: encontrada crescendo sobre rochas. Na Bahia, restrita à Chapada Diamantina, não tendo sido encontrada em outra região do estado.

Campylopus pilifer Brid., Mant. Musc. 72. 1819.

Descrição e ilustração: Frahm (1991).

Material examinado: BAHIA: Abaíra, Engenho dos Vieiras, beira do Rio Calado, $13^{\circ} 17^{\prime} \mathrm{S}$ e $41^{\circ} 52^{\prime} \mathrm{W}$, alt. $1150 \mathrm{~m}, 16 / 3 / 1992$, B. Stannard et al. s.n. (SP 284208); Mucugê, Unidade de manejo sustentável do projeto Sempre Viva, trilha do Garimpo, 17/3/1998, M.T.S. Stradmann s.n (ALCB 37733).

Comentários: encontrada crescendo sobre rochas. Na Bahia, restrita à Chapada Diamantina, não tendo sido encontrada em outra região do estado. 
Campylopus savannarum (C. Muell.) Mitt., J. Linn. Soc. Bot. 12: 85. 1869.

Descrição e ilustração: Frahm (1991).

Material examinado: BAHIA: Lençóis, 19/11/1983, C. Bastos 2 (ALCB 18254); Abaíra, Campo de Ouro Fino (alto), $13^{\circ} 15^{\prime} \mathrm{S}$ e $41^{\circ} 54^{\prime} \mathrm{W}$, alt. 1700 m, 19/1/1992, D.J.N. Hind \& R.F. Queiroz s.n. (SP 284209).

Comentários: encontrada crescendo sobre solo arenoso. Comum na Bahia, ocorrendo em cerrado e restinga.

Campylopus surinamensis C. Muell., Linnaea 21: 186.1848.

Descrição e ilustração: Frahm (1991).

Material examinado: BAHIA: Abaíra, Campo de Ouro Fino (alto), $13^{\circ} 15^{\prime} \mathrm{S}$ e $41^{\circ} 54^{\prime} \mathrm{W}$, alt. $1700 \mathrm{~m}$, 19/1/1992, D.J.N. Hind \& R.F. Queiroz s.n. (SP 284210).

Comentários: encontrada crescendo sobre o solo. Comum na Bahia, ocorrendo em mata mesófila secundária, cerrado e em áreas antrópicas.

Holomitrium arboreum Mitt., J.Linn. Soc. London Bot. 12: 58. 1869.

Descrição e ilustração: Sharp et al. (1994).

Material examinado: BAHIA: município de Abaíra, Campo de Ouro Fino (baixo), $13^{\circ} 15^{\prime} \mathrm{N}, 41^{\circ} 54^{\prime} \mathrm{W}$, alt. 1600-1700 m, 18/1/1992, D.J.N. Hind \& R.F. Queiroz s.n. (SP 284211); Mucugê, Unidade de Manejo Sustentável do projeto Sempre Viva, Cachoeira da Piabinha, Rio Piabinha, 18/3/1998, M.T.S. Stradmann s.n. (ALCB 37726).

Comentários: encontrada crescendo sobre tronco de árvore e rochas úmidas. Na Bahia, restrita à Chapada Diamantina, encontrada, também, em floresta de galeria.

\section{Leucobryaceae}

Leucobryum albicans (Schwaegr.) Lindb., Öfvers K. VetenskAkad. Foer. 20: 402. 1863.

Ilustração: Churchill \& Linares (1995).

Material examinado: BAHIA: Morro do Chapéu, $11^{\circ} 39^{\prime} \mathrm{S}$ e $41^{\circ} 07^{\prime} \mathrm{W}, 17 / 5 / 1978$, D.M. Vital 8027 (SP 146802).

Comentários: sem referência de substrato. Encontrada, também, em floresta de galeria.
Leucobryum albidum (Brid. ex P. Beauv.) Lindb., Öfvers K. VetenskAkad. Foer. 20: 402. 1863.

Descrição e ilustração: Sharp et al. (1994).

Material examinado: BAHIA: Rio de Contas, Pico das Almas, vertente leste, Campo do Queiroz, perto da trilha da Fazenda Silvina, 1332'S e $41^{\circ} 57^{\prime} \mathrm{W}$, alt. $1500 \mathrm{~m}, 27 / 11 / 1988$, R.M. Harley \& D.J. Hind 26637 (SP 229606).

Comentários: encontrada crescendo sobre rochas e solo arenoso. Na Bahia, restrita à Chapada Diamantina, não tendo sido encontrada em outra região.

*Leucobryum clavatum Hampe var. brevifolium Broth., Denkschr. Akad. Wiss. Wien Math. Nat. Kl. 83: 277. 1926.

Descrição e ilustração: Yano (1992).

Material examinado: BaHIA: Rio de Contas, Pico das Almas, vertente leste, Campo do Queiroz, perto da trilha da Fazenda Silvina, $13^{\circ} 32^{\prime} \mathrm{S}$ e $41^{\circ} 57^{\prime} \mathrm{W}$, alt. $1500 \mathrm{~m}, 27 / 11 / 1998$, R.M. Harley 26637 (ALCB 28247; CEPEC 55119).

Comentários: encontrada crescendo sobre tronco de árvore. Até o momento, não encontrada em outra região do estado.

Leucobryum crispum C. Muell., Syn. Musc. 1: 78. 1849.

Descrição e ilustração: Sharp et al. (1994).

Material examinado: BAHIA: Morro do Chapéu, 25/8/1970, D.V. Martins s.n. (ALCB 18198); Rio de Contas, Pico das Almas, vertente leste, Campo do Queiroz, $13^{\circ} 32^{\prime} \mathrm{S}$ e $41^{\circ} 57^{\prime} \mathrm{W}$, alt. $1500 \mathrm{~m}$, 11/11/1988, R.M. Harley et al. 26381 (SP 229608); Vale Pequeno, na margem leste do Campo de Queiroz, $13^{\circ} 32^{\prime}$ 'S e $41^{\circ} 56^{\prime} \mathrm{W}$, alt. $1500 \mathrm{~m}, 23 / 11 / 1988$, R.M. Harley et al. 26246 (SP 229607); Água Quente, Pico das Almas, vertente oeste, $13^{\circ} 31^{\prime} \mathrm{S}$ e $42^{\circ} 00^{\prime} \mathrm{W}$, alt. 1140 m, 17/12/1988, R.M. Harley \& B. Stannard 27562 (SP 229609); Abaíra, Campo de Ouro Fino (baixo), $13^{\circ} 15^{\prime} \mathrm{S}$ e $41^{\circ} 54^{\prime} \mathrm{W}$, alt. $1600-1700 \mathrm{~m}$, 18/1/1992, D.J.N. Hind \& R.F. Queiroz s.n. (SP 284223); Vale do Capão, 20/9/1996, A. Cerqueira s.n. (ALCB 37731); Mucugê, área de grotão, 20/9/1998, M.L. Guedes s.n. (ALCB 37739).

Comentários: encontrada crescendo sobre o solo e em fendas de rochas. Na Bahia, restrita à Cha- 
pada Diamantina, não tendo sido encontrada em outra região do estado.

*Leucobryum giganteum C. Muell., Syn. Musc. 1: 79.1849.

Descrição e ilustração: Yano (1996).

Material examinado: BAHIA: Morro do Chapéu, 3/4/1976, D.M. Vital 6020 (SP 135055); 17/5/1978, D.M. Vital 8046 (SP 146814); Abaíra, Campo de Ouro Fino (baixo), $13^{\circ} 15^{\prime} \mathrm{S}$ e $41^{\circ} 54^{\prime} \mathrm{W}$, alt. 1600 1700 m, 18/1/1992, D.J.N. Hind \& R.F. Queiroz s.n. (SP 284224).

Comentários: encontrada crescendo sobre o solo. Até o momento, não encontrada em outra região do estado.

Leucobryum martianum (Hornsch.) Hampe, Linnaea 17: 317. 1843.

Descrição e ilustração: Bartram (1949); Sharp et al. (1994).

Material examinado: BAHIA: Abaíra, Campo de Ouro Fino (baixo), $13^{\circ} 15^{\prime} \mathrm{S}$ e $41^{\circ} 54^{\prime} \mathrm{W}$, alt. 16001700 m, 18/1/1992, D.J.N. Hind \& R.F. Queiroz s.n. (SP 284225).

Comentários: encontrada crescendo sobre o solo. Comum na Bahia, ocorrendo em cerrado.

*Leucobryum sordidum Ängstr., Öfvers K. VetenskAkad. Foerh. 33(4): 7. 1877.

Descrição e ilustração: Yano (1992).

Material examinado: BAHIA: Lençóis, Rio Mucugezinho, ca. $1000 \mathrm{~m}$ alt., próximo ao morro do Pai Inácio, 20/12/1984, B. Stannard et al. s.n. (SP 222558).

Comentários: encontrada crescendo sobre tronco de árvore e rocha. Até o momento, não encontrada em outra região do estado.

Ochrobryum gardneri (C. Muell.) Lindb., Act. Soc. Sci. Fenn. 10: 229. 1872.

Descrição e ilustração: Sharp et al. (1994).

Material examinado: BAHIA: Morro do Chapéu, 3/4/1976, D.M. Vital 6021 (SP 135056).

Comentários: encontrada crescendo sobre tronco de árvore. Pouco comum na Bahia, citada apenas por Allen (1992), sem referência de localidade.
Octoblepharum albidum Hedw., Spec. Musc. 50. 1801.

Descrição e ilustração: Bartram (1949); Sharp et al. (1994).

Material examinado: Bahia: Lençóis, Serra do Pai Inácio, 11/7/1973, A.L. Costa s.n. (ALCB 18195).

Comentários: encontrada crescendo sobre o solo. Espécie de mais ampla distribuição na Bahia, ocorrendo em floresta ombrófila, floresta estacional, floresta de galeria, cerrado, caatinga, restinga, mata mesófila secundária e em áreas urbanas.

*Octoblepharum cocuiense Mitt., J. Linn. Soc. London Bot. 12: 109. 1869.

Descrição e ilustração: Yano (1992).

Material examinado: BAHIA: Morro do Chapéu, 4/4/1976, D.M. Vital 6054 (SP 135077).

Comentários: encontrada crescendo em fendas de rochas. Até o momento, não encontrada em outras regiões do estado.

\section{II - Hepatophyta}

\section{Herbertaceae}

Herbertus divergens (Steph.) Herz., Hedwigia 31: 14. 1892.

Descrição e ilustração: Fulford (1963)

Material examinado: BAHIA: Abaíra, Campo de Ouro Fino (baixo), $13^{\circ} 15^{\prime} \mathrm{S}$ e $41^{\circ} 54^{\prime} \mathrm{W}$, alt. 16001700 m, 18/1/1992, D.J.N. Hind \& R.F. Queiroz s.n. (SP 284220; SP 284222); Riacho da Taquara, $13^{\circ} 15^{\prime} \mathrm{S}$ e $41^{\circ} 55^{\prime} \mathrm{W}$, alt. $1670 \mathrm{~m}, 14 / 2 / 1992$, B. Stannard et al. s.n. (SP 284221).

Comentários: encontrada crescendo sobre tronco de árvore e solo. Na Bahia, restrita à Chapada Diamantina, não tendo sido encontrada em outra região do estado.

\section{Trichocoleaceae}

*Trichocolea flaccida (Spruce) Jack. \& Steph., Hedwigia 31: 14. 1892.

Descrição e ilustração: Fulford (1963).

Material examinado: BAHIA: Abaíra, Campo de Ouro Fino (baixo), $13^{\circ} 15^{\prime} \mathrm{S}$ e $41^{\circ} 54^{\prime} \mathrm{W}$, alt. 16001700 m, 18/1/1992, D.J.N. Hind \& R.F. Queiroz s.n. (SP 284183). 
Comentários: encontrada crescendo sobre tronco de árvore. Até o momento, não encontrada em outra região do estado.

\section{Geocalycaceae}

*Lophocolea bidentula (Nees) Fulf., Mem. New York Bot. Gdn. 11(4): 439. 1966.

Descrição e ilustração: Fulford (1976)

Material examinado: BAHIA: Abaíra, Campo de Ouro Fino (baixo), $13^{\circ} 15^{\prime} \mathrm{S}$ e $41^{\circ} 54$ 'W, alt. 1600 $1700 \mathrm{~m}, 25 / 1 / 1992$, D.J.N. Hind \& R.F. Queiroz s.n. (SP 284182).

Comentários: encontrada crescendo sobre rocha. Até o momento, não encontrada em outra região do estado.

\section{Plagiochilaceae}

Plagiochila bahiensis Lindenb., Spec. Hepat. fasc. 5: 136. 1843.

Descrição e ilustração: Lindenberg (1844).

Material examinado: BAHIA: Mucugê, Unidade de manejo sustentável do projeto Sempre Viva, 18/3/1998, M.T.S. Stradmann s.n. (ALCB 37736); 20/9/1998, M.L. Guedes s.n. (ALCB 37743).

Comentários: encontrada crescendo sobre tronco de árvore. Citada para a Bahia por Lindenberg (1844), Gottsche et al. (1844) e Stephani (1905), mas sem referência de localidade.

*Plagiochila cf. biserialis Lehm. \& Lindenb., Spec. Hepat. fasc. 5: 126. 1843.

Descrição e ilustração: Lindenberg (1844).

Material examinado: BAHIA: Abaíra, Mata da Serra do Rei, $14^{\circ} 16^{\prime} \mathrm{S}$ e $41^{\circ} 54^{\prime} \mathrm{W}$, alt. 1550-1650 m, 17/2/1992, R.M. Harley et al. s.n. (SP 284195).

Comentários: encontrada crescendo sobre tronco de árvore. Até o momento, não encontrada em outra região do estado.

*Plagiochila corrugata (Nees) Nees \& Mont., Am. Sci. Nat. Bot. sér. 2,5: 52. 1836.

Descrição e ilustração: Lindenberg (1844).

Material examinado: BAHIA: Abaíra, Campo de Ouro Fino (baixo), $13^{\circ} 15^{\prime} \mathrm{S}$ e $41^{\circ} 54^{\prime} \mathrm{W}$, alt. 1600 1700 m, 18/1/1992, D.J.N. Hind \& R.F. Queiroz s.n. (SP 284196).
Comentários: encontrada crescendo na base do tronco de árvore. Até o momento, não encontrada em outra região do estado.

* Plagiochila hypnoides Willd. ex Lindenb., Spec. Hepat. fasc. 2,4: 37. 1840.

Descrição e ilustração: Lindenberg (1844).

Material examinado: BAHIA: Abaíra, Campo de Ouro Fino (baixo), $13^{\circ} 15^{\prime} \mathrm{S}$ e $41^{\circ} 54^{\prime} \mathrm{W}$, alt. 1600 1700 m, 25/1/1992, D.J.N. Hind \& R.F. Queiroz s.n. (SP 284197).

Comentários: encontrada crescendo sobre rochas. Até o momento, não encontrada em outra região do estado.

* Plagiochila rutilans Lindenb., Spec. Hepat. fasc. 2.4: 47. 1840.

Descrição e ilustração: Lindenberg (1844).

Material examinado: BaHIA: Abaíra, Campo de Ouro Fino (baixo), $13^{\circ} 15^{\prime} \mathrm{S}$ e $41^{\circ} 54^{\prime} \mathrm{W}$, alt. 1600 1700 m, 18/1/1992, D.J.N. Hind \& R.F. Queiroz s.n (SP 284198; SP 284199); 25/1/1992, D.J.N. Hind \& R.F. Queiroz s.n. (SP 284200).

Comentários: encontrada crescendo sobre tronco de árvore. Encontrada, também, em floresta ombrófila e floresta estacional.

\section{Lepidoziaceae}

*Bazzania nitida (Web.) Grolle, Revue Bryol. Lichénol. 29: 210. 1960.

Descrição e ilustração: Fulford (1963).

Material examinado: BAHIA: Abaíra, Riacho da Taquara, $13^{\circ} 15^{\prime} \mathrm{S}$ e $41^{\circ} 55^{\prime} \mathrm{W}$, alt. $1670 \mathrm{~m}$, 14/2/1992, B. Stannard et al. (SP 284191); Campo do Cigano, $13^{\circ} 15^{\prime} \mathrm{S}$ e $41^{\circ} 55^{\prime} \mathrm{W}$, alt. $1780 \mathrm{~m}$, 14/2/1992, R.M. Harley et al. s.n. (SP 284192).

Comentários: encontrada crescendo em fenda de rocha. Até o momento, não encontrada em outra região do estado.

*Bazzania stolonifera (Sw.) Trevis., Mem. R. Ist. Lombardo Cl. Sci. ser. 3, 4: 415. 1877.

Descrição e ilustração: Fulford (1963).

Material examinado: BAHIA: Abaíra, Riacho da Taquara, $13^{\circ} 15^{\prime} \mathrm{S}$ e $41^{\circ} 55^{\prime} \mathrm{W}$, alt. $1670 \mathrm{~m}$, 14/2/1992, B. Stannard et al. s.n. (SP 284193); Campo de Ouro Fino (baixo), $13^{\circ} 15^{\prime} \mathrm{S}, 41^{\circ} 54^{\prime} \mathrm{W}$, alt. 
$1600-1700$ m, 15/2/1992, R.M. Harley et al. s.n. (SP 284194).

Comentários: encontrada crescendo sobre o solo e tronco de árvore. Até o momento, não encontrada em outra região do estado.

Kurzia brasiliensis (Steph.) Grolle, Revue Bryol. Lichénol. 32: 175. 1963.

Descrição e ilustração: Fulford (1966).

Material examinado: BAHIA: Abaíra, Campo de Ouro Fino (baixo), $13^{\circ} 15^{\prime} \mathrm{S}$ e $41^{\circ} 54^{\prime} \mathrm{W}$, alt. 1600 1700 m, 25/1/1992, D.J.N. Hind \& R.F. Queiroz s.n. (SP 284185); 18/1/1992, D.J.N. Hind \& R.F. Queiroz s.n. (SP 284186); Riacho da Taquara, $13^{\circ} 15^{\prime} \mathrm{S}$ e $41^{\circ} 55^{\prime} \mathrm{W}$, alt. $1670 \mathrm{~m}, 14 / 2 / 1992$, B. Stannard et al. (SP 284187).

Comentários: encontrada crescendo sobre o solo e tronco em decomposição. Na Bahia, restrita à Chapada Diamantina.

Micropterygium reimersianum Herz., Hedwigia 81: 225. 1943.

Descrição e ilustração: Fulford (1966).

Material examinado: BAHIA: Abaíra, Riacho da Taquara, $13^{\circ} 15^{\prime} \mathrm{S}$ e $41^{\circ} 55^{\prime} \mathrm{W}$, alt. $1670 \mathrm{~m}$, 14/2/1992, B. Stannard et al. s.n. (SP 284243); Mucugê, Unidade de manejo sustentável do projeto Sempre Viva, 15/3/1998, M.T.S. Stradmann s.n. (ALCB 37725).

Comentários: encontrada crescendo sobre o solo. Na Bahia, restrita à Chapada Diamantina, não tendo sido encontrada em outra região do estado.

*Micropterygium trachyphyllum Reim., Hedwigia 73: 186. 1933.

Descrição e ilustração: Fulford (1966).

Material examinado: BaHIA: Abaíra, Campo de Ouro Fino (baixo), $13^{\circ} 15^{\prime} \mathrm{S}$ e $41^{\circ} 54^{\prime} \mathrm{W}$, alt. 1600 1700 m, 18/1/1992, D.J.N. Hind \& R.F. Queiroz s.n. (SP 284244); Riacho da Taquara, $13^{\circ} 15^{\prime} \mathrm{S}$ e $41^{\circ} 55^{\prime}$ W. alt. $1670 \mathrm{~m}, 14 / 2 / 1992$, B. Stannard et al. (SP 284245).

Comentários: encontrada crescendo sobre solo arenoso. Ocorre também em floresta ombrófila, na região sul do estado.

\section{Cephaloziaceae}

Nowellia curvifolia (Dicks.) Mitt., Nat. Hist. Azores 321. 1870.

Descrição e ilustração: Schuster (1980).

Material examinado: BAHIA: Abaíra, Riacho da Taquara, $13^{\circ} 15^{\prime} \mathrm{S}$ e $41^{\circ} 55^{\prime} \mathrm{W}$, alt. $1670 \mathrm{~m}$, 14/2/1992, B. Stannard et al. s.n. (SP 284242).

Comentários: encontrada na margem de riacho, em local sombreado. Na Bahia, restrita à Chapada Diamantina, não tendo sido encontrada em outra região do estado.

\section{Radulaceae}

* Radula macrostachya Lindenb. \& Gott. in Gottsche et al., Syn. Hepat. 726. 1847.

Descrição e ilustração: Oliveira e Silva (1998).

Material examinado: BAHIA: Abaíra, Mata da Serra do Rei, $14^{\circ} 16^{\prime} \mathrm{S}$ e $41^{\circ} 54^{\prime} \mathrm{W}$, alt. $1555-1650 \mathrm{~m}$, 17/2/1992, R.M. Harley et al. s.n. (SP 284188; SP 284189).

Comentários: encontrada crescendo sobre tronco de árvore. Até o momento, não encontrada em outra região do estado.

*Radula mexicana Lindenb. \& Gott., Mexik. Leverm. 150. 1863.

Descrição e ilustração: Oliveira e Silva (1998).

Material examinado: BAHIA: Abaíra, Campo de Ouro Fino (baixo), $13^{\circ} 15^{\prime} \mathrm{S}$ e $41^{\circ} 54^{\prime} \mathrm{W}$, alt. 1600 1700 m, 18/1/1992, D.J.N. Hind \& R.F. Queiroz s.n. (SP 284190).

Comentários: encontrada crescendo sobre tronco de árvore. Até o momento, não encontrada em outra região do estado.

\section{Frullaniaceae}

*Frullania beyrichiana (Lehm. \& Lindenb.) Lehm. \& Lindenb., Syn. Hepat. 460. 1845.

Descrição e ilustração: Stotler (1969).

Material examinado: BAHIA: Abaíra, Campo de Ouro Fino (baixo), $13^{\circ} 15^{\prime} \mathrm{S}$ e $41^{\circ} 54^{\prime} \mathrm{W}$, alt. 1600 1700 m, 25/1/1992, D.J.N. Hind \& R. F. Queiroz s.n. (SP 284217); Campo do Cigano, $13^{\circ} 15^{\prime} \mathrm{S}$ e $41^{\circ} 55^{\prime} \mathrm{W}$, alt. $1780 \mathrm{~m}, 14 / 2 / 1992$, R.M. Harley et al. s.n. (SP 284218).

Comentários: encontrada crescendo sobre rocha. Até o momento, não encontrada em outra região do estado. 


\begin{tabular}{|c|c|c|c|}
\hline Táxons & Chapada Diamantina & Serra da Piedade & Serra do Cipó \\
\hline \multicolumn{4}{|l|}{ I - Bryophyta } \\
\hline Bryum argenteum & $\mathrm{x}$ & $\mathrm{x}$ & \\
\hline Campylopus introflexus & $\mathrm{x}$ & & $\mathrm{x}$ \\
\hline Holomitrium arboreum & $\mathrm{x}$ & $\mathrm{x}$ & \\
\hline Leucobryum albicans & $\mathrm{x}$ & & $\mathrm{x}$ \\
\hline L. crispum & $\mathrm{x}$ & & $\mathrm{x}$ \\
\hline L. martianum & $\mathrm{x}$ & & $\mathrm{x}$ \\
\hline Octoblepharum albidum & $\mathrm{x}$ & & $\mathrm{x}$ \\
\hline O. cocuiense & $\mathrm{x}$ & & $\mathrm{x}$ \\
\hline Polytrichum juniperinum & $\mathrm{x}$ & $\mathrm{x}$ & $\mathrm{x}$ \\
\hline Sematophyllum subpinnatum & $\mathrm{x}$ & $\mathrm{x}$ & $\mathrm{x}$ \\
\hline Sphagnum magellanicum & $\mathrm{x}$ & & $\mathrm{x}$ \\
\hline Syrrhopodon prolifer var. tenuifolius & $\mathrm{x}$ & $\mathrm{x}$ & \\
\hline \multicolumn{4}{|l|}{ II - Hepatophyta } \\
\hline Kurzia brasiliensis & $\mathrm{x}$ & $\mathrm{x}$ & \\
\hline Total & 13 & 6 & 9 \\
\hline
\end{tabular}

*Frullania griffithsiana Gott., Syn. Hepat. 466. 1845.

Descrição e ilustração: Stotler (1969).

Material examinado: BAHIA: Abaíra, Campo do Cigano, $13^{\circ} 15^{\prime} \mathrm{S}$ e $41^{\circ} 55^{\prime} \mathrm{W}$, alt. $1780 \mathrm{~m}, 14 / 2 / 1992$,

R.M. Harley et al. s.n. (SP 284219).

Comentários: encontrada crescendo sobre o solo e em fenda de rocha. Até o momento, não encontrada em outra região do estado.

\section{Lejeuneaceae}

Brachiolejeunea leiboldiana (Gott. \& Lindenb.) Schiffn., Hedwigia 33: 182. 1894.

Descrição: Gradstein (1994).

Material examinado: BAHIA: Abaíra, Campo de Ouro Fino (baixo), $13^{\circ} 15^{\prime} \mathrm{S}$ e $41^{\circ} 54^{\prime} \mathrm{W}$, alt. 1600 1700 m, 15/2/1992, R.M. Harley et al. s.n. (SP 284239).

Comentários: encontrada crescendo sobre tronco de árvore. Referida por Gradstein (1994) para a Serra da Água de Rega, sem especificação do ecossistema.
Bryopteris diffusa (Sw.) Nees, Syn. Hepat. 286. 1845.

Descrição e ilustração: Stotler \& CrandallStotler (1974).

Material examinado: Bahia: Morro do Chapéu, $11^{\circ} 39^{\prime} \mathrm{S}$ e $41^{\circ} 07^{\prime} \mathrm{W}, 17 / 5 / 1978$, D.M. Vital 8040 (SP 133015).

Comentários: encontrada sobre tronco de árvore. Comum na Bahia, ocorrendo em floresta ombrófila, floresta estacional e restinga arbórea.

*Lejeunea cavifolia (Ehrh.) Lindenb. emend. Buch, Revue Bryol. Lichénol. 7: 240. 1935.

Descrição e ilustração: Schuster (1980).

Material examinado: BaHIA: Abaíra, Campo do Cigano, $13^{\circ} 15^{\prime}$ 'S e $41^{\circ} 55^{\prime} \mathrm{W}$, alt. $1780 \mathrm{~m}, 14 / 2 / 1992$, R.M. Harley et al. s.n. (SP 284240).

Comentários: encontrada crescendo sobre o solo e em fenda de rocha. Até o momento, não encontrada em outra região do estado.

Lejeunea flava (Sw.) Nees, Naturgesch. Eur. leberm. 3: 227. 1838.

Descrição e ilustração: Schuster (1980). 
Material examinado: BAHIA: Mucugê, 20/9/1998, M.L. Guedes s.n. (ALCB 37743).

Comentários: encontrada crescendo em fenda de rocha. Comum na Bahia, ocorrendo em floresta ombrófila, restinga arbórea e em áreas antrópicas.

Marchesinia brachiata (Sw.) Schiffn., Naturl. Pflanzenfam. 1(3): 128. 1893.

Descrição e ilustração: Gradstein (1994).

Material examinado: BAHIA: Abaíra, Campo de Ouro Fino (baixo), $13^{\circ} 15^{\prime} \mathrm{S}$ e $41^{\circ} 54^{\prime} \mathrm{W}$, alt. 1600 1700 m, 18/1/1992, D.J.N. Hind \& R.F. Queiroz s.n. (SP 284241).

Comentários: encontrada crescendo na base de tronco de árvore. Citada para a Bahia por Gradstein (1994), mas sem referência da localidade.

Neurolejeunea breutelii (Gott.) Evans, Bull. Torrey Bot. Club. 34: 13. 1907.

Descrição e ilustração: Gradstein (1994).

Material examinado: BAHIA: Abaíra, Campo de Ouro Fino (baixo), $13^{\circ} 15^{\prime} \mathrm{S}$ e $41^{\circ} 54^{\prime} \mathrm{W}$, alt. 1600 1700 m, 25/1/1992, D.J.N. Hind \& R.F. Queiroz s.n. (SP 284201); Mata da Serra do Rei, $14^{\circ} 16^{\prime}$ S e $41^{\circ} 54^{\prime} \mathrm{W}$, alt. $1555-1650 \mathrm{~m}, 17 / 2 / 1992$, R.M. Harley et al. s.n. (SP 284202).

Comentários: encontrada crescendo sobre o solo, rocha e tronco de árvore. Comum na Bahia, ocorrendo também em floresta ombrófila, no sul do estado.

\section{Discussão}

Os campos rupestres, bem como toda a região da Chapada Diamantina, devido às suas peculiares condições físico-climáticas, com períodos de alta umidade atmosférica e relevo característico, propiciam uma variedade de microhabitats que abrigam uma flora briofítica especializada e bem diversificada. Muitas das espécies aí encontradas são típicas dessa região, não ocorrendo em outros locais do estado da Bahia, a exemplo de Aptychopsis pyrrhophylla, Campylopus julaceus, Holomitrium arboreum, espécies de Polytrichum, Pyrrhobryum spiniforme, Schlotheimia rugifolia, dentre as Bryophyta, e os gêneros Bazzania, Herbertus, Kurzia, Trichocolea, algumas espécies de Plagiochila e Nowellia curvifolia, dentre as Hepatophyta. Com- parando-se com os dados de Yano (1987), para a Serra do Cipó e Yano \& Carvalho (1995), para a Serra da Piedade, ambas em Minas Gerais, pode-se verificar algumas espécies em comum (Tabela 1). Tais espécies, exceto Bryum argenteum e Sematophyllum subpinnatum, são características de campos rupestres ou florestas de galeria, típicas de regiões montanhosas.

Algumas famílias são bem representadas na região da Chapada Diamantina podendo destacar-se Dicranaceae, Orthotrichaceae, Sphagnaceae e Leucobryaceae, dentre as Bryophyta e Lepidoziaceae, Plagiochilaceae, Herbertaceae e Trichocoleaceae, entre as Hepatophyta, sendo que essas duas últimas só ocorreram na Chapada Diamantina.

Os dados obtidos a partir desse e dos demais trabalhos realizados nessa Chapada, no estado da Bahia, quando confrontados com trabalhos feitos em outras regiões montanhosas do país, irão permitir a elaboração de um inventário das briófitas de habitats montanhosos do Brasil, o que possibilitará uma melhor visão fitogeográfica das espécies brasileiras.

Agradecimentos - Os autores são gratos à Bióloga Maria Theresa S. Stradmann pela realização de coletas na área da Chapada Diamantina e ao Dr. André Maurício de Carvalho, Curador do Herbário do CEPEC, pelo envio de duplicatas ao Herbário ALCB.

\section{Referências bibliográficas}

ALLEN, B.H. 1992. A revision of Ochrobryum (Leucobryaceae). Contributions from the University of Michigan Herbarium 18:113-130.

ALLEN, B.H. \& CROSBY, M.R. 1986. Revision of the genus Squamidium (Musci: Meteoriaceae). The Journal of the Hattori Botanical Laboratory 61:423-476.

BARTRAM, E.B. 1949. Mosses of Guatemala. Fieldiana Botany 25:1-442.

BASTOS, C.J.P., STRADMANN, M.T.S. \& VILAS BÔASBASTOS, S.B. 1998. Additional contribution to the bryophyte flora of the Chapada Diamantina National Park, State of Bahia, Brazil. Tropical Bryology 15:15-20.

BUCK, W.R. 1998. Pleurocarpous mosses of the West Indies. Memoirs of the New York Botanical Garden 82:1-400.

CEI - Centro de Estatística e Informações. 1994. Informações básicas dos municípios baianos: Região Chapada Diamantina. Secretaria de Planejamento, Governo do estado da Bahia, Salvador.

CHURCHILL, S.P. \& LINARES C.E.L. 1995. Prodomus Bryologiae Novo-Granatensis. Introduccion a la flora de musgos de Colombia. Biblioteca Jose Jeronimo Triana 12:1-924.

CRUM, H. 1984. North American Flora. Sphagnopsida. Sphagnaceae. The New York Botanical Garden, New York. 
CRUM, H. 1987. New species of Sphagnum from South America. Journal of the Hattori Botanical Laboratory 63:77-97.

FARIAS, H.C. 1984. A família Polytrichaceae no Rio Grande do Sul, Brasil. I. Taxonomia. Iheringia, sér. Botânica 32:77-89.

FRAHM, J.-P. 1991. Dicranaceae: Campylopodioideae, Paraleucobryoideae. Flora Neotropica Monograph 54:1-237.

FULFORD, M.H. 1963. Manual of the leafy Hepaticae of Latin America. Part I. Memoirs of the New York Botanical Garden 11:1-172.

FULFORD, M.H. 1966. Manual of the leafy Hepaticae of Latin America. Part II. Memoirs of the New York Botanical Garden 11:173-276

FULFORD, M.H. 1976. Manual of the leafy Hepaticae of Latin America. Part IV. Memoirs of the New York Botanical Garden 11:393-535.

GOTTSCHE, C.M., LINDENBERG, J.B.G. \& NEES VON ESENBEEK, C.G. 1844. Synopsis Hepaticarum. J.A. Messmer Ec., Hamburger.

GRADSTEIN, S.R. 1994. Lejeuneaceae: Ptychanthoideae, Brachiolejeuneae. Flora Neotropica Monograph 62:1-216.

HARLEY, R.M. 1995a. Introduction. In Flora of the Pico das Almas, Chapada Diamantina - Bahia, Brazil (B. L. Stannard, ed.). Royal Botanic Garden, Kew, p.1-40.

HARLEY, R.M. 1995b. Bryophyta. In Flora of the Pico das Almas, Chapada Diamantina - Bahia, Brazil (B.L. Stannard, ed.). Royal Botanic Garden, Kew, p.803-812.

LINDENBERG, J.B.G. 1844. Monographia Hepaticarum. Generis Plagiochilae. Sumptibus Henry \& Cohen, Bonn.

MÜLLER, C. 1851. Synopsis muscorum frondosorum omnium hucusque cognitorum 2. Musci vegetations pleurocarpieae. Sumptibus Alb. Foerstner, Berlim.

OCHI, H. 1980. A revision of the Neotropical Bryoideae, Musci. The Journal of the Faculty of Education Tottori University, Natural Science 29:49-154.

OLIVEIRA E SILVA, M.I.M.N. 1998. Briófitas da Reserva Ecológica do Rio da Pedra, município de Mangaratiba, do Parque Estadual da Ilha Grande e da Reserva Biológica Estadual da Praia do Sul, município de Angra dos Reis, Rio de Janeiro. Tese de doutorado, Universidade de São Paulo, São Paulo.

REESE, W.D. 1993. Calymperaceae. Flora Neotropica Monograph 58:1-102.

SCHUSTER, R.M. 1980. The Hepaticae and Anthocerotae of North America east of the Hundredth Meridian. Columbia University Press, New York, v. 4.
SCHUSTER, R.M. 1984. Evolution, phylogeny and classification of the Hepaticae. In New Manual of Bryology (R.M. Schuster, ed.). The Hattori Botanical Laboratory, Nichinan, v.2, p.892-1070.

SHARP, A.J., CRUM, H. \& ECKEL, P. 1994. The Moss Flora of Mexico. Memoirs of the New York Botanical Garden 69:11113.

STEPHANI, F. 1905. Species Hepaticarum. Bulletin de l'Herbier Boissier 5:351-366.

STOTLER, R.E. 1969. The genus Frullania subgenus Frullania in Latin America. Nova Hedwigia 18:357-555.

STOTLER, R.E. \& CRANDALL-STOTLER, B. 1974. A monograph of the genus Bryopteris (Swartz) Nees von Esenbeek. Bryophytorum Bibliotheca 3:1-159.

VITT, D.H. 1984. Classification of Bryopsida. In New manual of Bryology (R.M Schuster, ed.). The Hattori Botanical Laboratory, Nichinan, v. 2, p.696-759.

YANO, O. 1981. A Checklist of Brazilian Mosses. Journal of the Hattori Botanical Laboratory 50:279-456.

YANO, O. 1984. A Checklist of Brazilian Liverworts and Hornworts. Journal of the Hattori Botanical Laboratory 56:481-548.

YANO, O. 1987. Briófitas. In Flora da Serra do Cipó, Minas Gerais: caracterização e lista de espécies (A.M. Giulietti, N.L. Menezes, J.R Pirani, M. Meguro \& M.G.L Wanderley, eds.). Boletim de Botânica da Universidade de São Paulo 9, p.11-12.

YANO, O. 1989. An additional checklist of Brazilian Bryophytes. Journal of the Hattori Botanical Laboratory 66:371-434.

YANO, O. 1992. Leucobryaceae (Bryopsida) do Brasil. Tese de doutorado, Universidade de São Paulo, São Paulo.

YANO, O. 1995. A new additional annotated checklist of Brazilian Bryophytes. Journal of the Hattori Botanical Laboratory 78:137-182.

YANO, O. 1996. Criptógamos do Parque Estadual das Fontes do Ipiranga, São Paulo, SP. Briófitas, 1: Mniaceae, Rhizogoniaceae, Racopilaceae, Phyllogoniaceae e Leucobryaceae (Bryales). Hoehnea 23:81-98.

YANO, O. \& BASTOS, C.J.P. 1994. Musgos do estado da Bahia, Brasil. Biologica Brasilica 6:9-26.

YANO, O. \& CARVALHO, A.B. 1995. Briófitas da Serra da Piedade, Minas Gerais, Brasil. In Anais do $9^{\underline{0}}$ Congresso da Sociedade Botânica de São Paulo:15-25.

YANO, O., PIRANI, J.R. \& SANTOS, D.P. 1985. O gênero Sphagnum (Bryopsida) nas regiões sul e sudeste do Brasil. Revista Brasileira de Botânica 8:55-80. 